\title{
SPECTRAL SENSING RESEARCH FOR SURFACE AND AIR MONITORING IN CHEMICAL, BIOLOGICAL AND RADIOLOGICAL DEFENSE AND SECURITY APPLICATIONS
}




\section{Editor-in-Chief: $\quad$ M. S. Shur}

\section{Published}

Vol. 34: Radiation Effects and Soft Errors in Integrated Circuits and Electronic Devices eds. R. D. Schrimpf and D. M. Fleetwood

Vol. 35: Proceedings of the 2004 IEEE Lester Eastman Conference on High Performance Devices ed. Robert E. Leoni III

Vol. 36: Breakdown Phenomena in Semiconductors and Semiconductor Devices M. Levinshtein, J. Kostamovaara and S. Vainshtein

Vol. 37: Radiation Defect Engineering Kozlovski V. and Abrosimova V.

Vol. 38: Design of High-Speed Communication Circuits ed. R. Harjani

Vol. 39: High-Speed Optical Transceivers eds. Y. Liu and H. Yang

Vol. 40: SiC Materials and Devices - Vol. 1 eds. M. S. Shur, S. Rumyantsev and M. Levinshtein

Vol. 41: Frontiers in Electronics Proceedings of the WOFE-04 eds. H Iwai, Y. Nishi, M. S. Shur and H. Wong

Vol. 42: Transformational Science and Technology for the Current and Future Force eds. J. A. Parmentola, A. M. Rajendran, W. Bryzik, B. J. Walker, J. W. McCauley, J. Reifman, and N. M. Nasrabadi

Vol. 43: SiC Materials and Devices - Vol. 2 eds. M. S. Shur, S. Rumyantsev and M. Levinshtein

Vol. 44: Nanotubes and Nanowires ed. Peter J. Burke

Vol. 45: Proceedings of the 2006 IEEE Lester Eastman Conference on Advanced Semiconductor Devices eds. Michael S. Shur, P. Maki and J. Kolodzey

Vol. 46: Terahertz Science and Technology for Military and Security Applications eds. Dwight L. Woolard, James O. Jensen, R. Jennifer Hwu and Michael S. Shur

Vol. 47: Physics and Modeling of Tera- and Nano-Devices eds. M. Ryzhii and V. Ryzhii

Vol. 48: Spectral Sensing Research for Water Monitoring Applications and Frontier Science and Technology for Chemical, Biological and Radiological Defense eds. D. Woolard and J. Jensen 


\section{SPECTRAL SENSING RESEARCH FOR SURFACE AND AIR MONITORING IN CHEMICAL, BIOLOGICAL AND RADIOLOGICAL DEFENSE AND SECURITY APPLICATIONS}

Editors

Jean-Marc Theriault

Defence Research \& Development, Canada

\section{James O. Jensen}

US Army Edgewood Chemical Biological Center, USA 


\title{
Published by
}

World Scientific Publishing Co. Pte. Ltd.

5 Toh Tuck Link, Singapore 596224

USA office: 27 Warren Street, Suite 401-402, Hackensack, NJ 07601

UK office: 57 Shelton Street, Covent Garden, London WC2H 9HE

\section{British Library Cataloguing-in-Publication Data}

A catalogue record for this book is available from the British Library.

\section{Selected Topics in Electronics and Systems - Vol. 49 \\ SPECTRAL SENSING RESEARCH FOR SURFACE AND AIR MONITORING \\ IN CHEMICAL, BIOLOGICAL AND RADIOLOGICAL DEFENSE AND SECURITY APPLICATIONS}

Copyright @ 2009 by World Scientific Publishing Co. Pte. Ltd.

All rights reserved. This book, or parts thereof, may not be reproduced in any form or by any means, electronic or mechanical, including photocopying, recording or any information storage and retrieval system now known or to be invented, without written permission from the Publisher.

\begin{abstract}
For photocopying of material in this volume, please pay a copying fee through the Copyright Clearance Center, Inc., 222 Rosewood Drive, Danvers, MA 01923, USA. In this case permission to photocopy is not required from the publisher.
\end{abstract}

ISBN-13 978-981-283-591-8

ISBN-10 981-283-591-1

Editor: Tjan Kwang Wei

Printed in Singapore. 


\section{FOREWORD}

This special journal issue contains select papers from the 2006 International Symposium on Spectral Sensing Research (2006 ISSSR) that fall into the subject matter areas of Multispectral/Hyperspectral Techniques for Surface \& Air Monitoring in Chemical, Biological and Radiological (CB\&R) Defense Applications. The specific focus of the 2006 ISSSR was on the creation of new technology-program oriented networks that will serve as a research and development foundation for the advancement of the state-of-theart in spectroscopic-based early-warning sensor capabilities. In recent years, spectral sensing has experienced rapid technical advancement that has led to practical field sensors. Therefore spectral-based techniques exhibit the clear potential for providing more effective, economical and supportable (i.e., reagentless) solutions to military and homeland defense early-warning monitoring requirements for water, surface and air related sensing applications.

An increased emphasis on reagentless spectroscopy is motivated primarily by performance issues associated with traditional chemical and biological (CB) point and standoff techniques. In particular, sensors have been previously developed and fielded that rely heavily on reagents and/or burdensome support structures that are expensive and difficult to maintain and that have serious false alarm issues. Previously implemented technologies include biological assays, mass spectrometry and ion mobility. Other explored methodologies include novel materials (mips, smart ligands, amino acid sequences, aptamers, sol gel, aerogel, electro-conducting polymers, etc.) or bulk property interactions (electrochemistry, surface acoustic wave, surface plasmon resonance, thermal capacity) and combinations of the two.

At this time, extensive expertise exists in the multispectral/hyperspectral community for applications such as airborne and space-based sensing and imaging which has proved effective in monitoring weather, resource management (agriculture, forestry), oil/mineral deposits and CB detection in air releases. Hence, spectral-based techniques clearly have potential for providing near to mid-term solutions for many of the monitoring problems associated with CB\&R contaminations of water, surfaces and air. However, the ultimate realization of such spectroscopic techniques will probably require the fusion of many types of spectral-sensing techniques and modality. Therefore, standoff and point interrogation sensors are now sought that can provide for extremely high confidence in $\mathrm{CB} \& \mathrm{R}$ detection and monitoring scenarios and the goal of the 2006 ISSSR and this companion special issue is to organize and focus the science and technology base towards these important challenges. 
This special issue begins with a collection of research and development papers on the subject of spectroscopic and lidar techniques for "Surface Sensing and Monitoring" that have relevance to a general array of $C B \& R$ defense and security applications. The first section of papers focuses on novel scientific techniques and phenomenology applications that offer the potential for enhancing the effectiveness of existing hyperspectral/multispectral surface sensing methodologies. Examples of these research and development efforts include: Creation of reference data for vapors and liquids infrared spectral signatures suitable for quantitative analyses in environment monitoring; Novel approach for passive standoff detection of surface contaminants by differential polarization FTIR spectrometry, which mitigates sky radiance drifts and favours unambiguous contaminant detections; Advances in principal components analysis for the detection and classification of organic and organophosphorus analytes on soil from reflection-absorption spectroscopy; Novel developments in the detection of invisible bacilli spores on surfaces using a portable surface-enhanced raman spectroscopy (SERS) analyzer, which provides identification of chemicals based on their unique spectrum; Novel concept and simulation of a multiple-field-of-view (mfov) lidar for the standoff determination of bioaerosol size based on double scattering measurements; Advances in infrared spectrometry and analysis for the detection and differentiation of spore and vegetative forms of bacillus spp; New application of passive standoff radiometry for the measurement of explosives with a field result at a standoff distances of $60 \mathrm{~m}$; Investigation of signature and signal generation for explosive detection using thz timedomain spectroscopy which addresses the selectivity of thz spectra to distinguish the clutter from background spectra; Recent advances and results in differential passive Long Wave Infrared (LWIR) radiometry for the detection, identification and quantification of toxic chemical vapor clouds in an open-air environment; Comparative analyses of spectral background statistics in direct and differential Fourier Transform (LWIR) measurements, which serve to optimize spectral detection methods of atmospheric contaminants;

The second portion of the "Surface Sensing and Monitoring" section focuses on advanced sensing technology and algorithms developments that will impact spectroscopic-based sensing in the future. Some examples of these research and development efforts include: Overview of novel techniques under investigation by NVESD on active imaging of hard targets; Presentation of a high-resolution 2D imaging laser radar for occluded hard target viewing and identification with verification of system performance during a variety of operating conditions; Emerging technology of 3D flash Ladar focal planes and time dependent imaging that shows evidences and applications of this technology; Presentation of the design for the advanced responsive tacticallyeffective military imaging spectrometer (ARTEMIS); Assessment of the effects of image segmentation on subspace-based and covariance-based detection of anomalous sub-pixel materials in hyperspectral visible/near-IR/SWIR imagery; Advanced design of a spectral processing method for laser-induced fluorescence from threatening biological aerosols with simulations showing a good signal-to-background discrimination level; 
Development of a processing method for improving pixel purity index for endmember extraction in hyperspectral imagery; Novel methodology of signal processing for multicomponent Raman spectra of particulates for improving identification of chemical fingerprints; Development of support vector classification method for land cover and benthic habitat from hyperspectral images; and, Construction of a compact high peak power eye-safe optical parametric oscillator obtained by pumping with a master oscillator power amplifier.

The second section of this special issue contains a collection of research and development papers on the subject of spectroscopic techniques for "Air Sensing and Monitoring". The goal of this portion of the special issue is to investigate technologies for early warning, detection, and identification of chemical and biological contaminants in the atmosphere. This part examines optically-based sensors that are capable of detecting and identifying contaminants in the air. This area is divided into two categories: (1) point technologies where the sensor is in physical contact with the threat, and (2) standoff technologies where a sensor is physically separated from the threat by some distance. Active standoff techniques require the propagation of a probe beam such as in LIDAR systems. Passive standoff techniques rely on ambient electromagnetic radiation for detection and do not utilize probe beams.

New and novel spectroscopic techniques for detection, identification, and quantification of contaminants are examined. All regions of the electromagnetic spectrum, from radio waves to x-rays, are considered to be of interest. New spectral methods for the discrimination of contaminants from atmospheric interferents are of interest. Methods that increase detection sensitivity while reducing false alarms are also examined. Fluorescence, Raman, infrared, and terahertz spectroscopy are possible detection techniques locating and quantifying airborne chemical and biological contaminants.

Wide area detection and surveillance involves the simultaneous monitoring of the atmosphere over large surface areas for a possible contaminant. Spectral imaging techniques provide continuous, real-time monitoring of large areas for pollutions or other contaminants. Hyperspectral and ultraspectral imaging systems allow passive spatial/spectral monitoring of large areas which contain a possible pollution source. Hyperspectral and ultraspectral imaging systems also allow point source detection of hazards and determination of the flux of pollutants at important temporal and spatial scales Airborne and ground-based sensors are possible platforms for deployment of sensor.

Remote sensing of atmospheric pollutants usually involves scenarios with low signalto-noise. New and novel signal processing techniques are required in order to extract the pollutant's spectroscopic signatures. Radiometric models are very important in designing new sensors. Good models allow one to computationally prototype and test a sensor before actually building it.

New excitation sources for optical detection are examined. New laser sources for CB detection are relevant. Also better sources in other regions of the electromagnetic 
spectrum, such as far infrared and millimeter wave regions, are also being sought. Recent developments in IR source technologies have expanded the possibilities for creating chemical and biological sensors which are compact, highly selective, and extremely sensitive. These IR source technologies are e.g. Quantum Cascade Lasers, Optical Parametric Oscillators, Difference Frequency Generation.

Finally, the organizing committee 2006 ISSSR and the Editors of this special issue would like to recognize the following Best Paper Presentations that lead off this collection of technical papers:

Infrared Spectral Signatures: Creation of Reference Data for Vapors and Liquids

By Steven Sharpe, Pacific Northwest National Laboratory

Passive Standoff Detection of Surface Contaminants: A Novel Approach by Differential Polarization FTIR Spectrometry

By Jean-Marc Theriault, DRDC-Valcartier, Canada

Background Contributions in Direct and Differential Fourier Transform LWIR Measurements: A Comparative Analysis

By Francois Bouffard, DRDC-Valcartier, Canada

\section{Signal Processing of Multi-Component Raman Spectra of Particulate Matter \\ By Javier Foshesatto, University of Alaska, Fairbanks}

Computed Tomographic Imaging Spectrometer (CTIS) and a Snapshot Hyperspectral Imager and Polarimeter

By John Hartke, United States Military Academy

Wide Area Spectrometric Bioaerosol Monitoring in Canada: from SINBAHD to Biosense

By Jean-Robert Simard, DRDC-Valcartier, Canada

\section{Editors}

Jean-Marc Thériault, Defense Research \& Development, Canada James Jensen, U.S. Army Edgewood Chemical Biological Center 


\section{CONTENTS}

Foreword

\section{Surface Sensing \& Monitoring Sessions}

Infrared Spectral Signatures: Creation of Reference Data for Vapors and Liquids

S. Sharpe, T. Johnson, R. Sams, J. Hylden, J. Kleimeyer and

B. Rowland

Passive Standoff Detection of Surface Contaminants: A Novel Approach by Differential Polarization FTIR Spectrometry

J.-M. Thériault, H. Lavoie, E. Puckrin and F. Bouffard

Background Contributions in Direct and Differential Fourier Transform LWIR Measurements: A Comparative Analysis

F. Bouffard and J.-M. Thériault

Signal Processing of Multicomponent Raman Spectra of Particulate Matter

J. Fochesatto and J. Sloan

Signature and Signal Generation Aspects of Explosive Detection Using Terahertz Time-Domain Spectroscopy

R. Osiander, M. J. Fitch, M. Leahy-Hoppa, Y. Dikmelik and

J. B. Spicer

Novel Application of Passive Standoff Radiometry for the Measurement of Explosives

E. Puckrin, J.-M. Thériault, H. Lavoie, D. Dubé and P. Brousseau

Detection and Classification of Organic and Organophosphorus Analytes on Soil from Reflection-Absorption Spectroscopy

T. A. Blake, P. L. Gassman and N. B. Gallagher

Support Vector Classification of Land Cover and Benthic Habitat from Hyperspectral Images

V. Manian and M. Velez-Reyes

Some Effects of Image Segmentation on Subspace-Based and

Covariance-Based Detection of Anomalous Sub-Pixel Materials

C. Gittins, D. Konno, M. Hoke and A. Ratkowski 
Advanced Responsive Tactically-Effective Military Imaging Spectrometer (ARTEMIS) Design

T. W. Cooley, R. B. Lockwood, T. M. Davis, R. M. Nadile,

J. A. Gardner, P. S. Armstrong, Capt. A. M. Payton,

Capt. S. D. Straight, Lt. W. C. Henry, T. G. Chrien, E. L. Gussin and D. Makowski

Eyesafe Active Imaging of Hard Targets: An Overview of Techniques Under Investigation by NVESD

B. W. Schilling, S. R. Chinn, B. Thomas and T. J. Scholz

A High-Resolution 2D Imaging Laser Radar for Occluded Hard Target Viewing and Identification

R. J. Grasso, J. C. Wikman, D. P. Drouin, G. F. Dippel and P. I. Egbert

Three Dimensional Flash Ladar Focal Planes and Time Dependent Imaging

R. Stettner, H. Bailey and S. Silverman

Detection of Invisible Bacilli Spores on Surfaces Using a Portable

SERS-Based Analyzer

S. Farquharson and F. E. Inscore

Detection and Differentiation of Spore and Vegetative Forms of

Bacillus spp. Using Infrared Spectroscopic Methods

D. St. Amant, M. Campbell, A. Beck, L. Williams, J. Minter,

P. Collett, C. Zhu and A. Samuels

Spectral Processing of Laser-Induced Fluorescence from Threatening Biological Aerosols

P. Lahaie, J. R. Simard, J. Mcfee, S. Buteau, J. Ho, P. Mathieu, $G$. Roy and $V$. Larochelle

Standoff Determination of Bioaerosol Size Based on Double Scattering Measurement With MFOV Lidar; Concept and Numerical Simulation G. Roy and L. R. Bissonnette

Detection and Identification of Toxic Chemical Vapors in an Open-Air Environment by a Differential Passive LWIR Standoff Technique H. Lavoie, E. Puckrin and J.-M. Thériault

A Pyramid-Based Block of Skewers for Pixel Purity Index for Endmember Extraction in Hyperspectral Imagery C.-I. Chang, M. Hsueh, W. Liu, C.-C. Wu, F. Chaudhry, G. Solyar and A. Plaza 
A Compact Eye-Safe OPO Pumped by a Nd:YAG Microchip MOPA

J. Ding, B. W. Odom, A. R. Geiger and R. D. Richmond

\section{Air Sensing \& Monitoring Sessions}

Wide Area Spectrometric Bioaerosol Monitoring in Canada: From

SINBAHD to Biosense

J.-R. Simard, S. Buteau, P. Lahaie, P. Mathieu, G. Roy,

V. Larochelle, B. Dery, J. McFee and J. Ho

Computed Tomographic Imaging Spectrometer (CTIS) and a Snapshot Hyperspectral Imager and Polarimeter

J. Hartke, N. Hagan, B. A. Kinder and E. L. Dereniak

Hyperspectral Imaging Using Chromotomography: A Fieldable Visible Instrument for Transient Events

R. L. Bostick and G. P. Perram

Advanced Hyperspectral Algorithms for Tactical Target Detection and Discrimination

A. Schaum

AIRIS — The Canadian Hyperspectral Imager; Current Status and Future Developments

P. Fournier, T. Smithson and D. St-Germain

The Hypertemporal-Hyperspectral Analysis Test Station - HYHATS

T. Old, R. Hendrick, D. Higham, N. Palmer and C. Manning

Wavelength Selective Bolometer Design

S. Han, J.-Y. Jung and D. P. Neikirk

Multisensory Detection System for Damage Control and Situational Awareness

C. P. Minor, D. A. Steinhurst, K. J. Johnson,

S. L. Rose-Pehrsson, J. C. Owrutsky, S. C. Wales and D. T. Gottuk

Inexpensive Chemical Defense Network for a Fixed Site

J. A. Seeley, M. Angel, R. L. Aggarwal, T. H. Jeys,

A. Sanchez-Rubio, W. Dinatale and J. M. Richardson

Precision Measurement of Atmospheric Trace Constituents Using a

Compact Fabry-Perot Radiometer

W. S. Heaps, E. L. Wilson and E. M. Georgieva 
Background Characterization with a Scanned Fourier Transform Spectrometer

A. K. Lazarevich, D. A. Oursler and D. D. Duncan

Spectral Signatures of Acetone Vapor from Ultraviolet to Millimeter Wavelengths

R. E. Peale, A. V. Muravjov, C. J. Fredricksen, G. D. Boreman,

H. Saxena, G. Braunstein, V. L. Vaks, A. V. Maslovsky and

S. D. Nikifirov

The Standoff Aerosol Active Signature Testbed (SAAST) at MIT Lincoln Laboratory

J. M. Richardson and J. C. Aldridge

Discrimination Between Natural Dense Dust Clouds with IR Spectral Measurements

E. Agassi, A. Ronen, N. Shiloah and E. Hirsch

Signal Processing Algorithms for Staring Single Pixel Hyperspectral Sensors

D. Manolakis, M. Rossacci, E. O'Donnell, F. M. D'Amico

Performance Estimation Tools for: Decoupling by Filtering of Temperature and Emissivity (DEFILTE), An Algorithm for Thermal Hyperspectral Image Processing

P. Lahaie

Estimating the Limit of Bio-Aerosol Detection with Passive Infrared Spectroscopy

A. Ifarraguerri, A. Ben-David and R. G. Vanderbeek

Eye Safe Polarization Diversity LIDAR for Aerosol Studies: Concept Design and Preliminary Applications

J. Fochesatto, R. L. Collins, K. Sassen, H. Quantz and K. Ganapuram

Aerosol Type-Identification using UV-NIR-IR LIDAR System

S. Egert and D. Peri

Rare-Earth Doped Potassium Lead Bromide Mid-IR Laser Sources for Standoff Detection

K. C. Mandal, S. H. Kang, M. Choi and R. D. Rauh

3D Deconvolution of Vibration Corrupted Hyperspectral Images

A. H. Webster, M. R. Davenport and J.-P. Ardouin 